\title{
Crack detection in "as-cast" steel using laser triangulation and machine learning
}

\author{
J. Veitch-Michaelis*, Y. Tao*, D. Walton*, J-P. Muller*, B. Crutchley ${ }^{\dagger}$, J. Storey ${ }^{\dagger}$, C. Paterson ${ }^{\ddagger}$, A. Richardson ${ }^{\ddagger}$ \\ *Mullard Space Science Laboratory, University College London, Dorking, UK, RH5 6NT \\ Email: (j.veitchmichaelis.12, y.tao,d.walton,j.muller)@ucl.ac.uk \\ ${ }^{\dagger}$ IS-Instruments Ltd, Tonbridge, UK, TN9 1SP \\ Email: (bcrutchley, jstorey)@is-instruments.com \\ ${ }^{\ddagger}$ Materials Processing Institute, Eston Rd, Middlesbrough, UK, TS6 6US \\ Email: (chris.paterson, andy.richardson)@mpiuk.com
}

\begin{abstract}
We describe a high-accuracy inspection system designed to automatically detect cracks in "as-cast" steel slabs. Real-time slab inspection requires instrumentation capable of withstanding high temperatures above the steel surface as well as coping with the dirty and dusty environment present in a steel mill. Crack detection is also challenging due to the presence of oxidation scale on the slab surface. A bespoke laser triangulation system has been developed, providing images at 250 fps with a calibrated surface resolution of $97 \mu \mathrm{m}$ from a $1 \mathrm{~m}$ standoff distance. Cracks are detected using a combination of morphological detection and SVM classifier. Results are reported from laboratory testing and from extended trials at a production steel mill.
\end{abstract}

Keywords-laser triangulation, challenging environment, 3D reconstruction, crack detection;

\section{INTRODUCTION}

Continuous casting accounted for an estimate of over $96 \%$ of crude steel production worldwide in 2014 and over $98 \%$ of UK steel production ${ }^{1}$. In comparison to ingot casting, where molten steel is poured into a stationary mould, continuous casting produces sections of metal, typically slabs or billets, with indeterminate length. The resulting lengths of metal may then be processed into a variety of derivative products such as plates, construction beams, rail sections or tubes.

Due to the wide variety of thermal and mechanical stresses involved in continuous casting, there is a risk of cracking and other defects at almost every stage in the process [1]. If defects are found in a slab, the defective surface is removed in a process called scarfing. Manual inspection of each individual slab is labour intensive, offline and efficient manual detection requires experienced operators. The alternative, scarfing every slab as a precaution provides a firmer guarantee on product consistency, but is resource intensive and wasteful as most slabs are defect-free. Even scarfing does not guarantee defect-free steel as there may be control issues with the scarfing equipment [2]. Ideally, a defect detection system should be able to identify problems during the casting process before the steel has fully cooled.

\footnotetext{
${ }^{1}$ World Steel Association Steel Statistical Yearbook 2015, aggregate of 65 countries comprising $>99 \%$ of worldwide steel production.
}

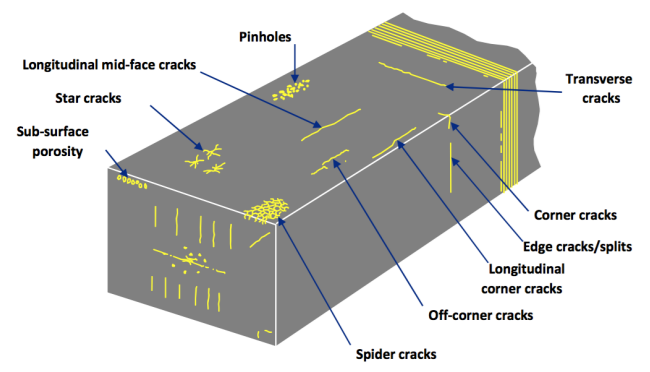

Figure 1. Major defect types found on "as-cast" semi-processed steel slab.

The TATA Scunthorpe plant sought a reduction in waste of 31,000 metric tons (t) per annum (1\% of annual plant yield, 2012) . Considering total TATA liquid steel production in the UK, (estimated at $8 \mathrm{Mt}$ per annum), a $1 \%$ yield improvement corresponds to 80,000 t per annum, worth $£ 1.77 \mathrm{M}$ assuming a value of $£ 30 / \mathrm{t}$ and also equates to a saving of $£ 4.5 \mathrm{M} \mathrm{CO}_{2}$ $\left(150,000 \mathrm{t} \mathrm{CO}_{2}\right)$.

The Innovate UK High Temperature Process Control (HTP-C) project investigated the possibility of using laser triangulation, in combination with high resolution optical imagery, for automated crack detection in "as-cast" steel. The developed instrument is capable of mapping the surface topology of hot moving steel at sub-mm resolution. These data are then passed to an algorithm that combines low level image processing with machine learning to detect cracks.

\section{A. Slab defects}

Figure 1, adapted from [1], shows a range of defects that can occur in continuously cast steel.

This project only considered surface defects as deep interior cracks are impossible to detect using visible wavelength light. Defects can range in size from sub-mm pinholes to mid-face cracks that may be several millimetres wide. Due to the presence of oxide scale on the steel surface [3], many of these defects are extremely difficult to detect in optical imagery alone. In as little as 30 seconds after exposure to oxygen-rich air, scale can form complex 3D structures on the slab surface such as blisters [4]. These structures do not necessarily indicate defective steel and 


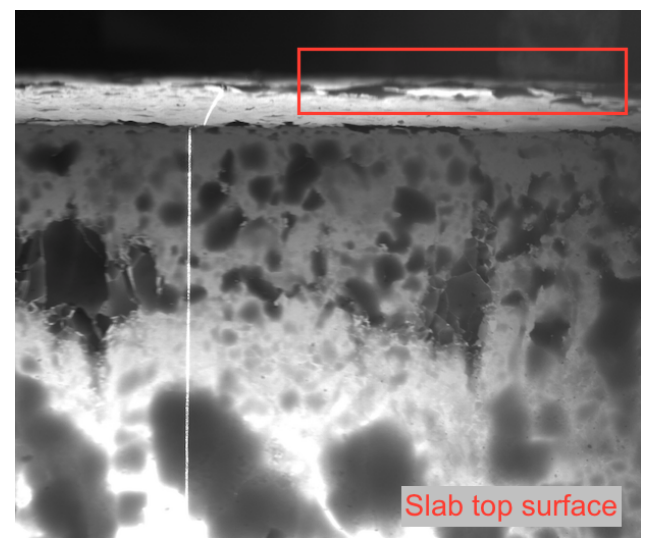

Figure 2. Continuously cast steel slab, imaged using the HTP-C instrument. The red box highlights scale blisters forming on the side of the slab. The laser stripe is visible in the left half of the image.

detection systems should be able to differentiate between scale features and slab defects [5]. Figure 2 shows a topdown view of a continuously cast steel slab, captured using the HTP-C instrument. The presence of scale is clearly visible as discolouration and $3 \mathrm{D}$ scale structures are visible on the side of the slab, such as the region marked by the red box.

\section{B. Related work}

General process inspection using both laser triangulation and optical imagery is well established [6], [7] and many COTS profiling systems are available. See, for example, products by Micro Epsilon ${ }^{2}$, MTI Instruments ${ }^{3}$ and LMI Technologies ${ }^{4}$. However most off-the-shelf 2D triangulation systems are not suitable for harsh environments.

As cracks are surface discontinuities, edge detectors are a natural choice for segmentation in images. Inspection systems for cast steel defect detection have been reported several times in the literature using morphological operations [8], [9], Wavelet [10] or Gabor filtering [11]. Both edge detection and morphological operations are fast and simple to implement on modern hardware. The main drawback to this approach is the lack of contextual information which is critical when analysing scale-contaminated surfaces.

Machine learning offers an alternative solution to this problem by allowing positive or negative feature classification. Martin et al. [12] proposed combining low-level edge detectors with a variety of machine learning techniques, from which logistical regression was found to have the best trade-off between speed, space and accuracy. Machine learning usually requires extensive training data which has historically been an issue for slab analysis [8], as there is little publicly available imagery to train on. Pernkopf [5],

\footnotetext{
${ }^{2}$ http://www.micro-epsilon.co.uk/laser-scanner/index.html

${ }^{3} \mathrm{http}: / /$ www.mtiinstruments.com/products/lasertriangulation.aspx

${ }^{4}$ http://lmi3d.com/products
}

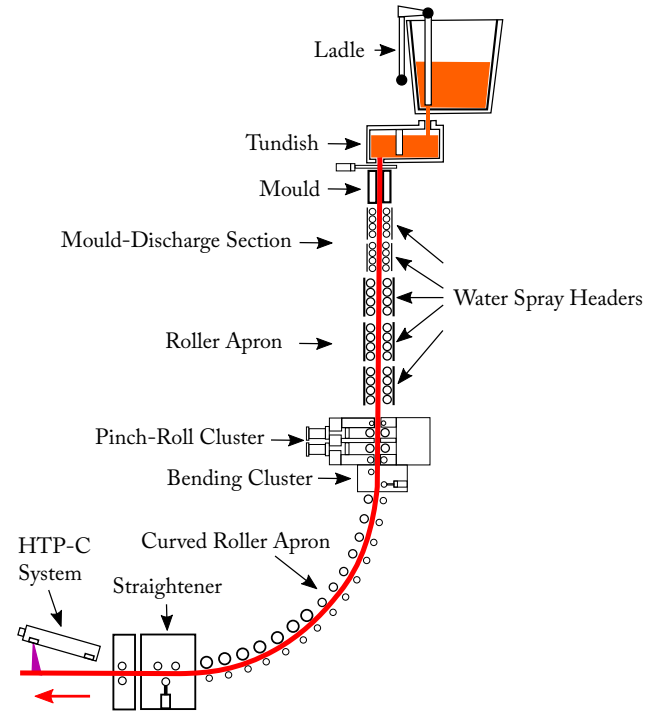

Figure 3. Typical horizontal-output continuous caster (not to scale). The HTP-C system was installed at the exit of the straightening rollers.

Table I

HTP-C INSTRUMENT GOAL REQUIREMENTS. THRESHOLD REQUIREMENTS SHOWN IN PARENTHESES.

\begin{tabular}{ll} 
Parameter & Specification \\
\hline Range measurement accuracy $(\mathrm{mm})$ & $0.1(0.3)$ \\
Depth of field $(\mathrm{mm})$ & $25(15)$ \\
Capture speed $(\mathrm{fps})$ & $250(150)$ \\
Spatial resolution at steel surface $(\mathrm{mm})$ & $0.1 \times 0.1(0.5 \times 0.5)$ \\
Size of features to be determined $(\mathrm{mm})$ & $0.1 \times 5(0.5 \times 10)$ \\
Field of view on steel & $300(100)$ \\
Laser & Eye safe
\end{tabular}

[13] suggested using laser triangulation to monitor (cold) milled steel blocks, avoiding intensity imaging entirely. After acquiring profile data, slab sections were classified using a Bayesian network with an accuracy of $98 \%$.

Landstrom and Thurley [9] applied morphological operations (off-line) to 3D profile data acquired using laser triangulation. The segmented cracks were then classified using logistical regression. They reported segmentation accuracy of over $80 \%$. Typical cracks in their data were several millimetres wide and up to $60 \mathrm{~mm}$ long.

Alvarez et al. [14] presented a system that has been used on-line at the ArcelorMittal plant in Avilas, Spain over a period of two years. The system used conoscopic holography to reliably detect defects over $100 \mathrm{~mm}$ in length, but a detailed description of the classification process was not provided.

\section{Measurement System}

\section{A. System requirements}

System requirements, agreed with TATA, are shown in Table I. It was assumed that the casting speed was $0.9 \mathrm{~m} / \mathrm{min}$ which, along with the resolution requirements, implies a 


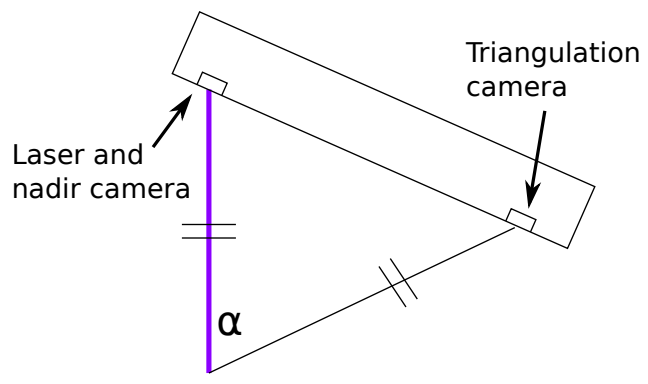

Figure 4. HTP-C system geometry. The system is angled such that the path length from the surface to each camera is the same.

minimum frame rate of $150 \mathrm{~Hz}(0.9 \mathrm{~m} / 0.1 \mathrm{~mm} / 60 \mathrm{~s})$. However at higher casting speeds, up to $1.5 \mathrm{~m} / \mathrm{min}$, a higher frame rate of $250 \mathrm{~Hz}$ is necessary.

The location for the instrument was chosen to be at the straightener exit as shown in Figure 3. This site allowed access to the instrument during plant downtimes and allowed imaging of the steel with as little scale as possible. Initial thermal measurements, suggested an air temperature in excess of $200{ }^{\circ} \mathrm{C}$ at a height of $1 \mathrm{~m}$ above the slab surface.

\section{B. Mechanical and optical design}

The HTP-C system consisted of a blue/violet $(405 \mathrm{~nm})$ laser stripe projector and associated triangulation camera. The system geometry is shown in Figure 4. Unlike a conventional laser triangulation system, HTP-C included an additional overhead (nadir) camera positioned at the laser beam exit, approximately coaxial with the beam. The triangulation camera is positioned along a baseline and angled towards the laser line. The nadir camera recorded full-resolution images with minimal perspective distortion.

The cameras employed were IDS (Obersulm, Germany) ${ }^{5}$ UI-3370CPs mated with Schneider-Kreuznach (RhinelandPalatinate, Germany $)^{6}$ Unifoc Modular lenses with an effective focal length of $80 \mathrm{~mm}$. The cameras have a nominal resolution of $2048 \times 2048 \mathrm{px}$. They were operated in a windowed mode to allow higher frame rates whilst sacrificing vertical field of view. The cameras were temporally synchronised using their onboard triggering capability.

The projected pixel size and depth resolution $\left(p_{x}, p_{y}, \Delta Z\right)$ is given by:

$$
\left(p_{x}, p_{y}, \Delta Z\right) \approx\left(\Delta P, \frac{\Delta P}{\tan \alpha}, \frac{\Delta P}{\sin \alpha}\right) .
$$

Where $\Delta P$ is the size of one pixel projected onto the steel. The chosen triangulation angle, $\alpha=45^{\circ}$, gave a depth resolution of $67 \mu \mathrm{m}$ and a projected pixel size of $97 \mu \mathrm{m}$ in both horizontal axes.

\footnotetext{
${ }^{5}$ https://en.ids-imaging.com/

${ }^{6}$ http://www.schneiderkreuznach.com
}

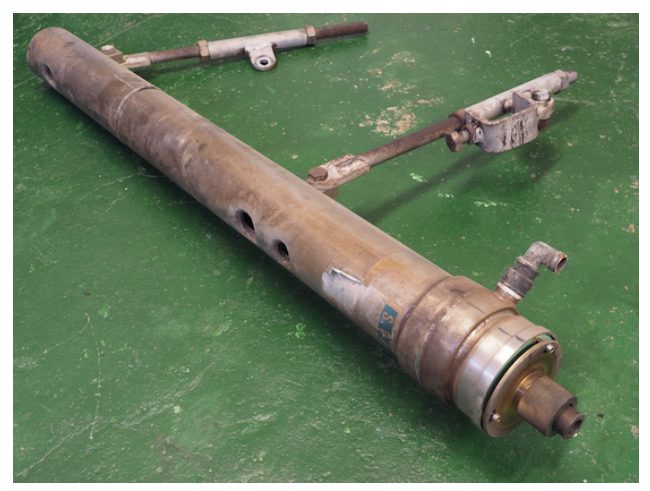

Figure 5. HTP-C cooled housing with apertures for two cameras and a laser stripe projector. The exterior shows extensive marking after being suspended above the caster for a period of several weeks.

The laser line generator was an Osela (Lachine, Canada) ${ }^{7}$ SL-405-35-S-C-15.0 operating at $405 \mathrm{~nm}$ with an output power of $35 \mathrm{~mW}$ and a fan angle of $10{ }^{\circ} \mathrm{C}$. This generator has a working range of $920-1000 \mathrm{~mm}$. Over the required depth of field, the laser line was expected to be less than $150 \mu \mathrm{m}$ wide on the steel.

Slabs exiting the straightener emit large amounts of infrared and near-infrared light. Dichroic blue filters manufactured by UQG Optics (Cambridge, UK) were fitted in front of the lenses. The filter cut off was at $480 \mathrm{~nm}$.

Due to the harsh environment, a custom cooled enclosure (Figure 5) was used to house the optical components. The housing was a modified furnace probe, commissioned from SVA Industrie Fernseh $\mathrm{GmbH}$ (Hilden, Germany) ${ }^{8}$, and featured a water-cooled outer sheath and internal air purging to prevent dust build-up. Three apertures were placed into the probe for the laser line generator and two cameras.

A rugged, fanless PC supplied by Logic Supply (Rijswijk, Netherlands) ${ }^{9}$ was used to control the cameras and run the crack detection software. The CPU was an Intel i7-3610QE with 4 physical cores running at $3.3 \mathrm{GHz}$.

\section{Robust laser line detection}

The problem of laser stripe detection is well known and several algorithm choices are available [15]. A simple centreof-mass peak detection method was chosen. Given a list of intensities and considering them in turn, if the first pixel number above the threshold is at $x_{1}$ and the last pixel above the threshold is at $x_{2}$ then the peak location, $x_{p}$ is simply $x_{p}=\left(x_{1}+x_{2}\right) / 2$.

Most stripe detection algorithms assume that the signal is not saturated and rely on a number of known-intensity points from which the peak centre is determined. This is not a valid assumption when imaging hot steel. Exposure

\footnotetext{
${ }^{7}$ http://www.osela.com

${ }^{8} \mathrm{http}: / /$ www.furnace-monitoring.tv/

${ }^{9}$ http://www.logicsupply.com/
} 


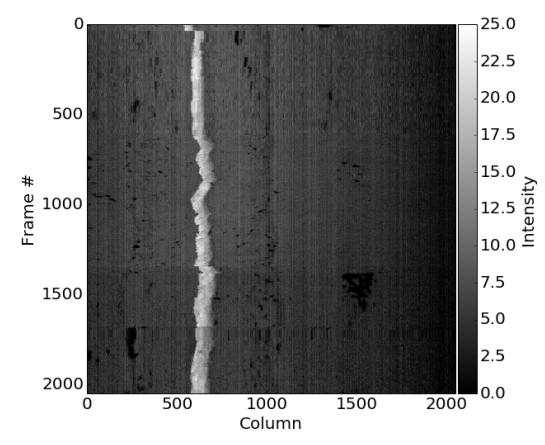

(a)

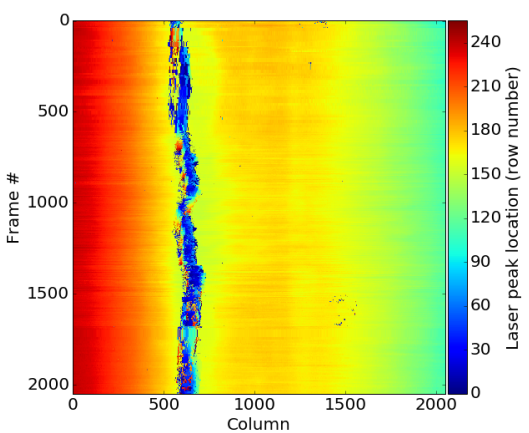

(b)

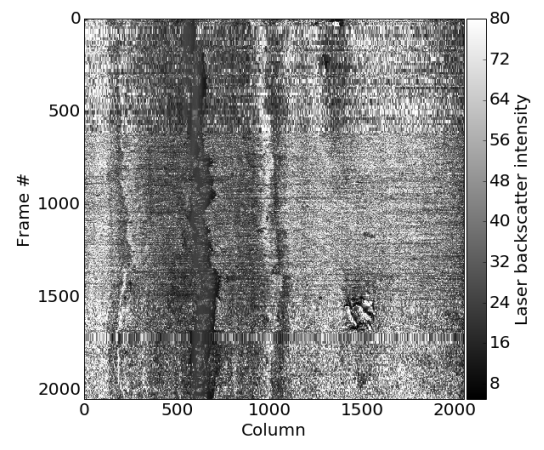

(c)

Figure 6. HTP-C data product example (a) Raw "linescan" image. (b) Uncalibrated laser peak location (c) Laser backscatter intensity. The frame contains a large longitudinal crack.

times were chosen to minimise laser overexposure, typically below $2 \mathrm{~ms}$, but saturation was still observed during casts. In practice this method was capable of accurately determining the laser line position even if saturation occured.

The threshold accuracy for spatial resolution was approximately 5 pixels on the steel surface. The width of the laser beam on the steel surface tended to be between 5-10 px. The algorithm was found to have an error of 1-2 px compared to a reference Gaussian fit. Storing sub-pixel peak locations was not performed due to storage considerations.

\section{Acquisition Software}

The minimum requirement of operation at $150 \mathrm{fps}$ implied that each frame must be acquired and processed within 6.7 $\mathrm{ms}$, the goal requirement of $250 \mathrm{fps}$ gave a frame processing time of $4 \mathrm{~ms}$. Operating the cameras in a windowed mode, with reduced vertical resolution of $256 \mathrm{px}$, allowed reliable image capture at up to $450 \mathrm{fps}$.

The acquisition software was developed in $\mathrm{C}++$ using a multithreaded, pipeline approach. Processing times of $<3$ ms per frame were achieved.

\section{E. Data Products}

Four data products were stored for each captured image: an internet synchronised UNIX timestamp, a row of raw image data, the laser line location for every column in the image and the height of the detected laser peak (backscatter intensity). The data for each frame was added to a stack. After a number of frames, the stack was stored to disk.

Examples of each stack of data products are shown in Figure 6. The steel image shown is a partial top surface view of a $300 \mathrm{~mm}$ wide mini-slab with a large centreline crack clearly present. The data were acquired at the Materials Processing Institute (MPI) on a 7 tonne pilot caster, primarily used for research.

The raw image data, Figure 6a, is formed from the first row in each image stacked over time to produce an image like that of a linescan camera. In this image, the crack appears brighter than the slab surface due to the exposed, hotter, slab interior. Figure $6 \mathrm{~b}$ shows the laser line location, a $2 \mathrm{D}$ representation of the topology of the surface. Note that there are a wide variety of surface features visible in the raw image which might be mistaken for defects. However, these regions are shown by the laser to be homogenous and mostly flat. Where the laser line falls on the crack, recovery of the peak is challenging and this is observed as missing data in the crack region. Finally the backscatter intensity image, Figure $6 \mathrm{c}$ provides insight into the reflectivity of the surface and allows monitoring for saturation of the laser line.

\section{F. Crack detection algorithm}

The crack detection algorithm was developed at UCL's Mullard Space Science Laboratory (MSSL). The algorithm combines texture feature based detection and supervised machine learning. Morphological and adaptive (Otsu) thresholding [16] were used in the first stage, texture feature based detection, and Support Vector Machine (SVM) was used as the classifier [17].

In the first stage, morphological edge detection and Otsu's adaptive thresholding combined with median and speckle filtering were used to detect potential cracks, labelled L0 (level 0) results. This first stage detection aimed to capture $100 \%$ of defects, regardless of false detection. Meanwhile, all potential cracks marked in the first stage were extracted and stored as a crack training database for supervised classification. In the second stage, an SVM classifier used the L0 results and user-selected training data (positive/negative samples) to output a refined list of cracks, labelled L1 (level 1). A flowchart of the processing pipeline is shown in Figure 7.

The crack detection process is per-pixel, so the output from the algorithm can be laid on top of the input imagery to be displayed to the user. The time to process a single $2048 \times 2048$ px data stack was 1.5 seconds. As this is less time than it takes to stack the data (4 seconds at $250 \mathrm{fps}$ ), the processing is effectively real-time. 


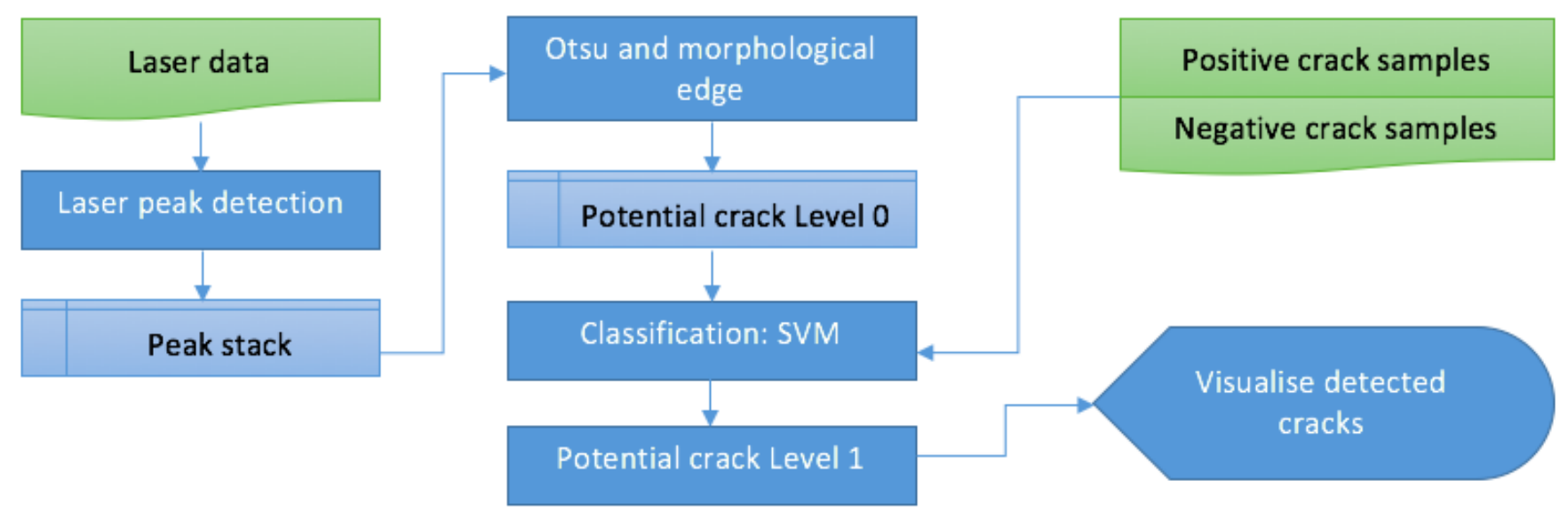

Figure 7. HTP-C crack detection algorithm flowchart

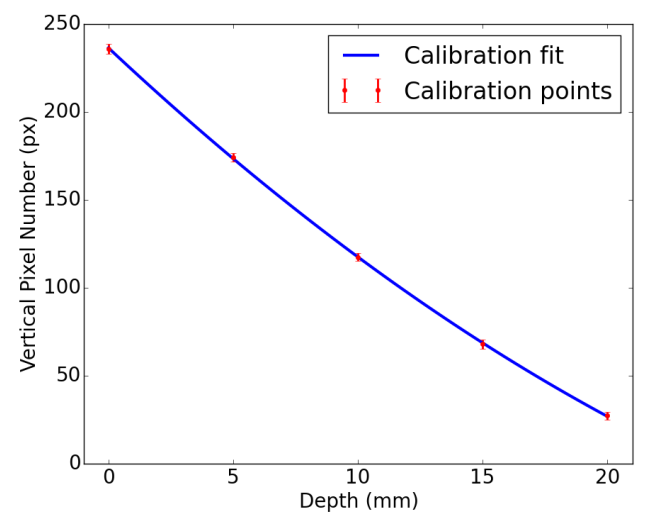

Figure 8. HTP-C calibration

\section{G. Calibration}

In order to produce a metric model of the steel surface, calibration was performed to convert line location in pixels to depth. The calibration was performed using a 3D stepped structure, printed using a Formlabs (Somerville, MA, USA) ${ }^{10}$ Form $1+$ stereo-lithography printer with a print resolution of $50 \mu \mathrm{m}$. The height between each step was $5 \mathrm{~mm}$.

The target was placed at the focus of the laser line and positioned so that 5 steps, corresponding to $25 \mathrm{~mm}$ of depth variation, were visible. A stack of 1280 frames of data was captured. The mean laser line location for each step was used as the calibration value. The data were fitted using a 2nd order polynomial. The intercept of the curve was not important as the point of zero-depth can be chosen arbitrarily. A plot of the calibration curve is shown in Figure 8.

The fit equation was $P=0.14 Z^{2}-13.29 Z+229.1$. Taking the derivative with respect to $Z$ and setting $Z=1 \mathrm{~m}$ gave a value of $\Delta Z / \Delta P=77 \mu \mathrm{m} / \mathrm{px}$. Considering the peak

\footnotetext{
${ }^{10} \mathrm{http}: / /$ formlabs.com/
}

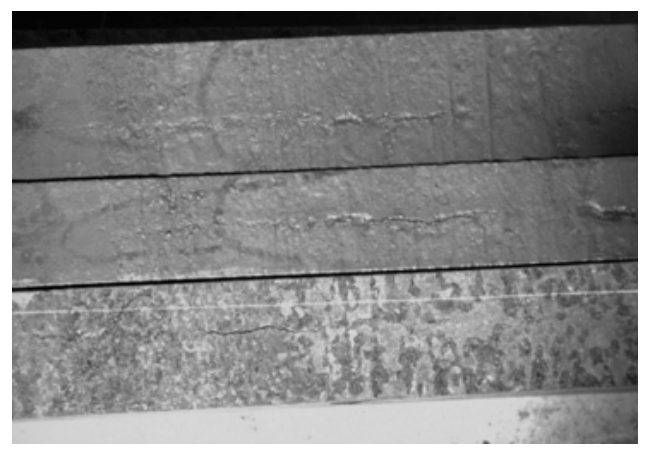

Figure 9. Defective steel samples placed on a carriage for imaging with the HTP-C instrument.

detection error of 1-2 px, the depth accuracy was therefore estimated to be between $77-144 \mu \mathrm{m} / \mathrm{px}$, within the threshold specification.

\section{Software VALIDATION}

A series of initial trials were performed at MSSL to evaluate the acquisition and crack detection software. Three samples of defective steel were provided by TATA Steel, shown in Figure 9.

The samples contained a variety of defects including longitudinal and semi-spider cracks. The instrument was positioned similarly to the expected configuration in the production steel plant. The samples were moved in a linear motion under the HTP-C instrument at a speed of $15 \mathrm{~mm} / \mathrm{s}$.

Figure 10 shows $\mathrm{L} 0$ and $\mathrm{L} 1$ crack detection results applied to the test images. Cracks are labelled as red pixels.

Although the smaller defects in the samples were not detected, the larger cracks were. The reason for this is likely due to under-exposure in the triangulation camera imagery. The crack detection algorithm relies on there being a large number of positive crack samples to train on. As more onsite trials are carried out, the training database will become more comprehensive and improve the true detection rate. 


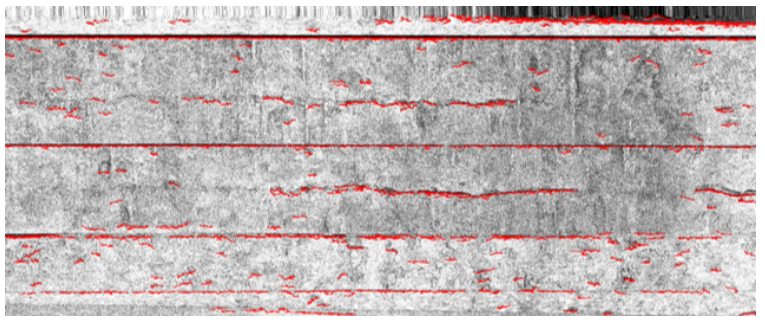

(a)

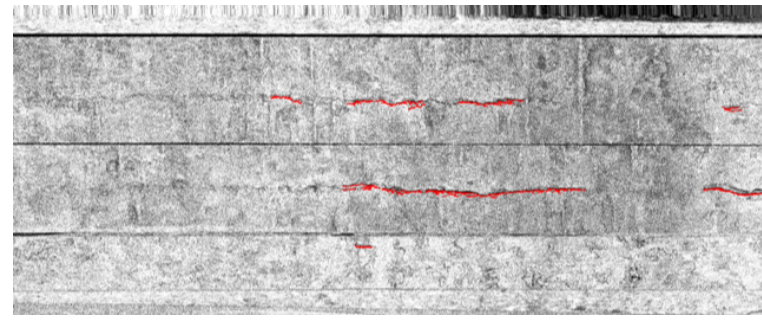

(b)

Figure 10. HTP-C crack detection algorithm applied to test imagery. (a) L0 detection results (b) L1 (refined) detection results. The vertical artefacts at the top of the image are due to acquisition while the slabs were stationary. Detected cracks are labelled as red pixels.

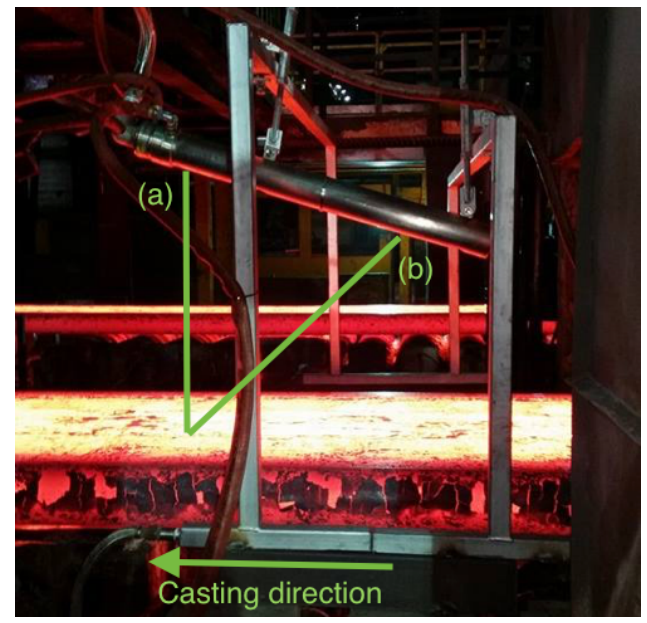

Figure 11. HTP-C system installed on a slab caster at the Concast facility at TATA Steel, Scunthorpe. (a) Nadir camera and laser. (b) Triangulation camera. Casting direction is from right to left as shown.

\section{FIELD TRIALS}

The HTP-C system was installed at the continuous casting (Concast) facility at TATA Steel, Scunthorpe, UK. The instrument was attached to a water-cooled metal frame which was spot-welded to a site near the first set of rollers. The acquisition PC was situated nearby behind a heat-resistant screen. Data and power cables were housed in an air-purged insulated sheath. Figure 11 shows the system in operation.

This image highlights a number of features of interest. First, there is a strong thermal gradient near the edge of the slab due to the increased surface area where heat is radiated away. Secondly, there is significant brightness variation on the upper surface of the slab due to scale. On the sides of the slab, there are several patches of scale which are peeling away or blistering as the steel cools.

The system was focused at a distance of $1 \mathrm{~m}$, coincident with the expected surface height of the slabs. The depth of focus is in shown in Figure 12. The backscatter intensity image shows the leading edge of a new slab. The object at the top of the image is the dummy bar, a piece of steel used to draw the new slab down from the mould. Below the

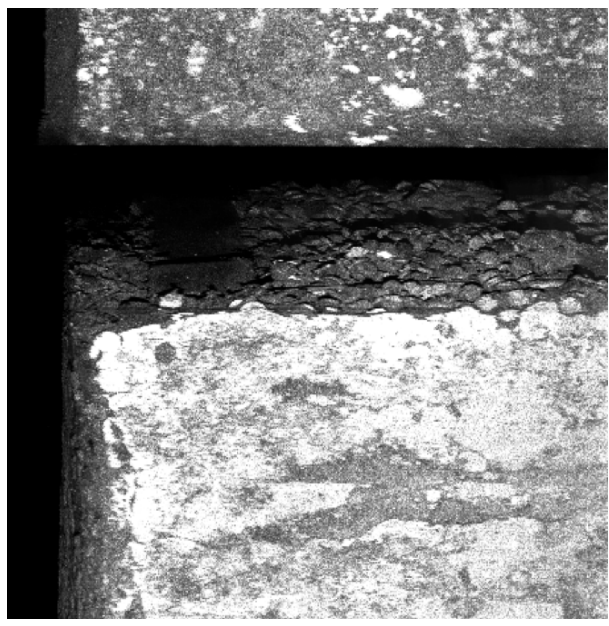

Figure 12. Laser backscatter image of the leading edge of a slab, acquired at the start of a cast. The dummy bar is visible at the top of the image with the slab below.

surface, the substructure of the slab is clearly visible.

Three widths of slabs were produced with this caster: $1610 \mathrm{~mm}, 1830 \mathrm{~mm}$ and $1970 \mathrm{~mm}$. Given that all three are routinely produced and the instrument was to be installed for a minimum of 6 weeks, with no possibility of removal, the instrument was adjusted such that useful data might be acquired in all three cases. In principle, the instrument could have been positioned to view the centre of the slab regardless of width. It was decided that viewing the edge of the slab would yield more useful data, since there was a wider variety of defects that might be visible. Figure 13 shows the fields of view for each slab width. The images were captured using the same exposure time of $1 \mathrm{~ms}$. When imaging $1970 \mathrm{~mm}$ slabs, the thermal gradient caused the images to saturate towards the centre of the slab. When imaging the wider slab widths, the laser module intermittently overheated and turned off.

\section{A. Results}

The instrument acquired data over several casts. Intermittent overheating of the laser module meant that, in some cases, only partial casts were imaged. Raw images were 


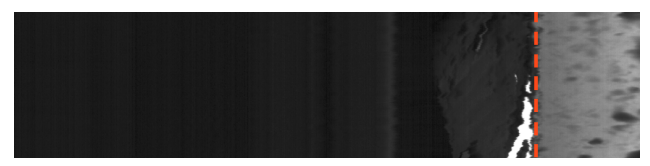

(a)

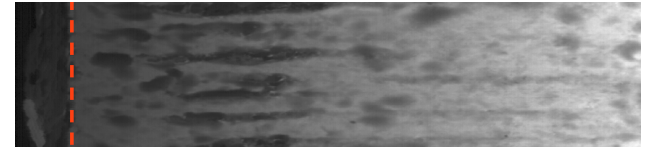

(b)

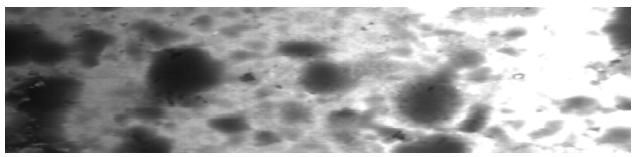

(c)

Figure 13. HTP-C instrument field of view for three slab widths at the Concast facility. (a) $1610 \mathrm{~mm}$ (b) $1830 \mathrm{~mm}$ (c) $1970 \mathrm{~mm}$. For (a) and (b), the slab edge is marked with a dashed line.

still captured regardless of the operational status of the laser. There was no indication of damage to the camera modules and the system remained in alignment throughout the trial.

The most promising results were from the $1610 \mathrm{~mm}$ width slabs as these did not cause overheating. Data from the $1830 \mathrm{~mm}$ width slabs were also acceptable when the laser was functioning. Data from the $1970 \mathrm{~mm}$ width slabs suffered from very high background intensities and were mostly unusable.

In almost all images, scale was visible. The effect on the data varied. In some cases it was possible to discern the scale topology. Figure 14 shows an example of this.

Close to the slab edge (left of image) the scale topology was captured, visible as intensity variations in the depth image. Towards the slab centre (right of image), the laser line is either not detectable by the system or is spuriously detected. This is a similar response to that expected of a crack, i.e. the laser line not being visible, so any classification result should also consider the shape of the feature.

Potential cracks were identified both by software and through manual analysis of the data. Figure 15 shows one example found via manual search (highlighted). There are prominent scale bubbles visible near the slab edge. Each bubble appears as if it is illuminated from one side. This effect is due to specular reflection of the laser beam rather than actual shadowing. The depth image clarifies that these features are raised compared to the surrounding surface, and hence confirmed as scale. However, further processing with the crack detection algorithm did not yield positive results. A typical example of L0 potential cracks detected in a different data stack is shown in Figure 15c.

The LO cracks are found in locations with strong edges, mostly around the edge of scale. The SVM classifier is able to correctly filter these as false positives.

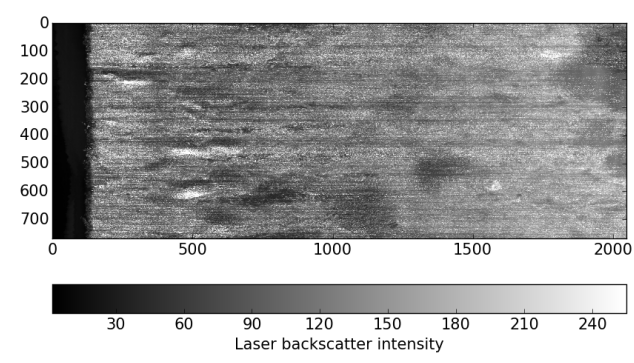

(a)

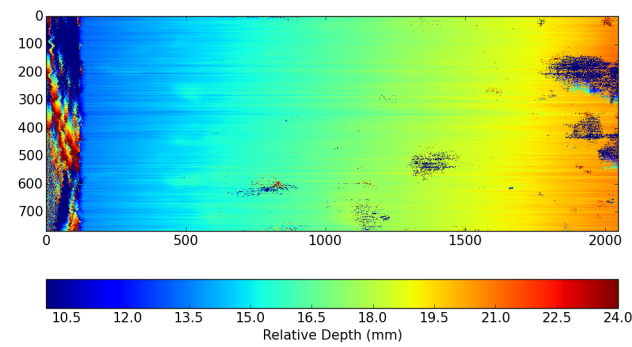

(b)

Figure 14. Effects of scale on (a) laser backscatter and (b) corresponding depth image.

The L0 results demonstrate the difficulty of detecting cracks directly using image edges only. The data returned from the instrument was hard for a naive algorithm to interpret due to the presence of scale. In the backscatter imagery, scale blistering appears as regions with strong intensity gradients. The detected edges caused scale to be mis-identified as potential cracks as we intended to further classify features in the L1 stage. In the depth data, particularly towards the middle of the slab, scale prevented detection of the laser line. In wide slabs, this region was often overexposed with the problem exacerbated by a strong thermal gradient across the slab. No cracks were discovered after the classification stage (L1).

An important cue for accurate defect detection is the relative depth of surface features. Scale, by definition, forms a layer above the surface of the steel and effects like blistering and bubbling also form above the surface. Depth information is therefore useful for robust filtering of potential defects.

\section{CONCLUSIONS}

This project demonstrated an inspection system capable of profiling and analysing hot "as-cast" steel in real time. The software recovers sub-mm surface topology, detects and verifies cracks using morphological operations and SVM classification. The crack detection algorithm was verified on ground truth samples provided by TATA steel. The instrument was installed at a production steel mill and captured data over several casts.

In real-world testing, no cracks were found after the second stage of filtering (L0 to L1). The lack of L1 cracks detected is not necessarily due to poor performance of the 


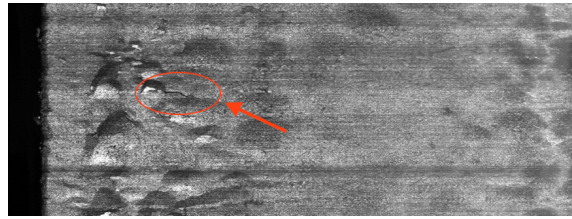

(a)

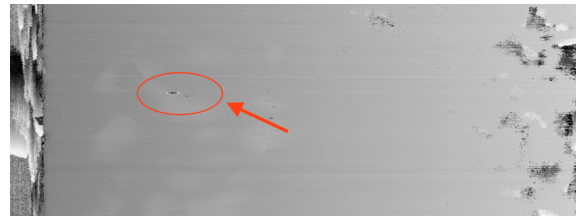

(b)

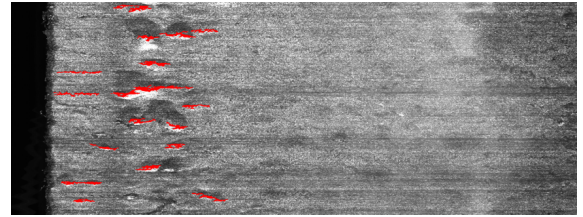

(c)

Figure 15. Manually identified potential crack (a) laser backscatter image (b) depth image. (c) Potential L0 cracks located using the crack detection algorithm before being filtered with the SVM classifier.

detection algorithm. It was only possible to acquire data over a sshort period of time and there was no indication from TATA Steel that any defective steel had been cast during testing.

Future work will aim to improve the quantity and quality of training data, as this is necessary for reliable crack detection. This may involve extensive manual ground truth generation.

\section{ACKNOWLEDGMENT}

The authors would like to thank the Centre for Process Innovation, the Materials Processing Institute and TATA Steel for providing access to production facilities for instrument testing, and Innovate UK (formerly the Technology Strategy Board) for funding this project.

\section{REFERENCES}

[1] J. K. Brimacombe and K. Sorimachi, "Crack formation in the continuous casting of steel," Metallurgical Transactions $B$, vol. 8, no. 2, pp. 489-505, 1977.

[2] S.-G. Ryu, D.-c. Choi, Y.-J. Jeon, S. J. Lee, J. P. Yun, and S. W. Kim, "Detection of Scarfing Faults on the Edges of Slabs," ISIJ International, vol. 54, no. 1, pp. 112-118, 2014.

[3] R. Y. Chen and W. Y. D. Yeun, "Review of the HighTemperature Oxidation of Iron and Carbon Steels in Air or Oxygen," Oxidation of Metals, vol. 59, no. 5-6, pp. 433-468, 2003.

[4] Y. Kondo, H. Tanei, N. Suzuki, K. Ushioda, and M. Maeda, "Blistering Behavior during Oxide Scale Formation on Steel Surface," ISIJ International, vol. 51, no. 10, pp. 1696-1702, 2011.

[5] F. Pernkopf, "3D surface acquisition and reconstruction for inspection of raw steel products," Computers in Industry, vol. 56, no. 8-9, pp. 876-885, Dec. 2005.

[6] T. S. Newman and A. K. Jain, "A Survey of Automated Visual Inspection," Computer Vision and Image Understanding, vol. 61, no. 2, pp. 231-262, 1995.

[7] E. N. Malamas, E. G. M. Petrakis, M. Zervakis, L. Petit, and J.-D. Legat, "A survey on industrial vision systems, applications and tools," IMAVIS, vol. 21, no. 2, pp. 171-188, Feb. 2003.
[8] B. R. Suresh, R. A. Fundakowski, T. S. Levitt, and J. E. Overland, "A Real-Time Automated Visual Inspection System for Hot Steel Slabs," Pattern Analysis and Machine Intelligence, IEEE Transactions on, vol. PAMI-5, no. 6, pp. 563-572, Nov. 1983.

[9] A. Landstrom and M. J. Thurley, "Morphology-Based Crack Detection for Steel Slabs," Selected Topics in Signal Processing, IEEE Journal of, vol. 6, no. 7, pp. 866-875, Aug. 2012.

[10] Y.-h. AI and K. XU, "Surface Detection of Continuous Casting Slabs Based on Curvelet Transform and Kernel Locality Preserving Projections," Journal of Iron and Steel Research, International, vol. 20, no. 5, pp. 80-86, May 2013.

[11] Y.-J. Jeon, D.-c. Choi, S. J. Lee, J. P. Yun, and S. W. Kim, "Defect detection for corner cracks in steel billets using a wavelet reconstruction method," Journal of the Optical Society of America A, vol. 31, no. 2, pp. 227-237, Feb. 2014.

[12] D. R. Martin, C. C. Fowlkes, and J. Malik, "Learning to detect natural image boundaries using local brightness, color, and texture cues," Pattern Analysis and Machine Intelligence, IEEE Transactions on, vol. 26, no. 5, pp. 530-549, May 2004.

[13] F. Pernkopf, "Detection of surface defects on raw steel blocks using Bayesian network classifiers," Pattern Analysis and Applications, vol. 7, no. 3, pp. 333-342, 2004.

[14] I. Alvarez, J. Marina, J. M. Enguita, C. Fraga, and R. Garcia, "Industrial online surface defects detection in continuous casting hot slabs," SPIE Europe ..., pp. 73 891X-73 891X-9, Jun. 2009.

[15] D. K. Naidu and R. B. Fisher, "A Comparative Analysis of Algorithms for Determining the Peak Position of a Stripe to Sub-pixel Accuracy," in British Machine Vision Conference 1991. Springer-Verlag London Limited, 1991, pp. 28.1-28.9.

[16] N. Otsu, "A Threshold Selection Method from Gray-Level Histograms," Systems, Man and Cybernetics, IEEE Transactions on, vol. 9, no. 1, pp. 62-66, 1979.

[17] J. A. K. Suykens and J. Vandewalle, "Least Squares Support Vector Machine Classifiers," Neural processing letters, vol. 9 , no. 3, pp. 293-300, 1999. 